Fürstenhaus Liechtenstein - Böhmische Länder - Fürstentum Liechtenstein

Ad honorem Thomas Winkelbauer 


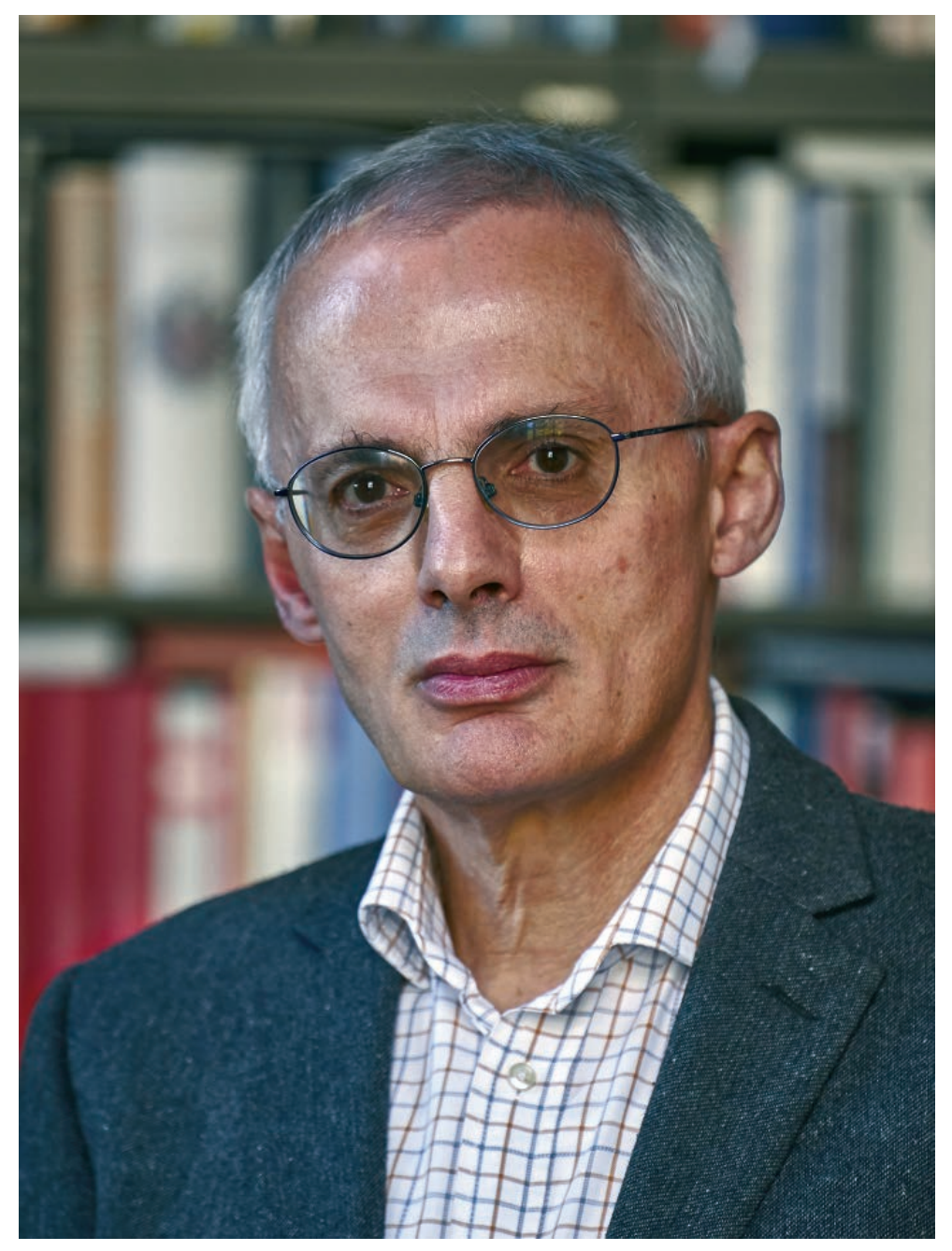

Thomas Winkelbauer 


\title{
Thomas Winkelbauer und die Erforschung der Geschichte des Fürstenhauses Liechtenstein
}

\section{Thomas Winkelbauer and the Research of the Princely House Liechtensteins History}

Tomáš Knoz / knoz@phil.muni.cz

Historický ústav, Filozofická fakulta, Masarykova univerzita, Brno, CZ

\begin{abstract}
Profesor Thomas Winkelbauer of the University of Vienna is a respected historian of the Early Modern Age. His bibliography reveals that a large part of his research work focuses on the princely Liechtenstein family, both in his monographs (in particular Gundaker of Liechtenstein and Karl of Liechtenstein), as well as contextually (as part of the history of the aristocracy in the Early Modern Age and the wider context of Austrian history). Thomas Winkelbauer wrote an important monograph on Gundaker of Liechtenstein, to which he later added specialist studies and editorial sources. He uses this Austrian-Moravian to demonstrate how the world of the aristocracy worked in the Early Modern Age. He also examines the themes of religion and conversion as one of the decisive elements directly linked to the political rise of individuals and families in the Early Modern Age. It is also important to remember that for Winkelbauer, the Liechtensteins represent "the aristocracy which knows no bounds". All of these issues are also addressed in Winkelbauer's outstanding synthesis on Austrian history in the 16th and 17th centuries.
\end{abstract}

\section{Keywords}

Thomas Winkelbauer, Princely House Liechtenstein, history, early new age, Gundaker of Liechtenstein, Austria 
Als Thomas Winkelbauer am 14. März 2017 seinen 60. Geburtstag beging, wurden wiederholt auch seine Verdienste bei der Erforschung der österreichischen Geschichte, der politischen und sozialen Strukturen der Frühen Neuzeit, zugleich aber auch der "grenzüberschreitenden Geschichte“ sowie der Geschichte der Historiographie hervorgehoben. Wenn wir einen Blick in Winkelbauers Bibliographie werfen, können wir feststellen, dass viele dieser Themen und methodologischen Herangehensweisen so oder ähnlich auch die Geschichte des Hauses Liechtenstein berühren und dass Persönlichkeiten aus dem Umfeld des gleichnamigen Fürstenhauses in dieser oder jener Weise eine wichtige bzw. am Ende sogar entscheidende Rolle auch in Winkelbauers Monographien und wegweisenden Aufsätzen spielen, die die einzelnen Etappen seiner bisherigen Forschungstätigkeit umrahmen.

Das Forschungsinteresse Thomas Winkelbauers konzentriert sich natürlich nicht ausschließlich auf die Geschichte des Hauses Liechtenstein, sondern ist vielmehr in einem ziemlich breiten Kontext angelegt, und die Geschichte des Adelsgeschlechts wird hier vor dem Hintergrund der Geschichte des frühneuzeitlichen Adels als Repräsentant der zeitgenössischen Eliten präsentiert, auf der anderen Seite zugleich auch mit Hilfe von Methoden der Sozialgeschichte und in Gegenüberstellung mit Untersuchungen des Verhältnisses zwischen der Aristokratie und deren Untertanen. Eine ganze Reihe dieser Beziehungen spielte sich auf der Basis rechtlicher Normen und finanzieller Transaktionen ab, was ein weiteres Interessensgebiet in den Forschungen Winkelbauers darstellt. Thomas Winkelbauer hat mehrere Untersuchungen zur führenden Persönlichkeit des Hauses Liechtenstein in der Frühen Neuzeit und ganz allgemein in der Geschichte vorgelegt, und zwar zu Karl von Liechtenstein. Dessen ungeachtet nimmt den wichtigsten Platz seines Interesses Karls jüngster Bruder Gundaker ein. Diesem hat Winkelbauer - ausgehend vom Text seiner Habilitationsschrift - eine seiner bedeutsamsten Monographien gewidmet und dabei anhand dessen Persönlichkeit alle grundlegenden, die sich formende Persönlichkeit eines frühneuzeitlichen Aristokraten ausmachenden Merkmale herausgestellt. An erster Stelle muss in diesem Zusammenhang an die religiöse Seite der Persönlichkeit erinnert werden (die, wie zahlreiche deutsche Historiker unterstreichen, als Teil der Frühen Neuzeit immerhin ja ein „konfessionelles Zeitalter“ darstellte), und vor allem Konvertierungen vom Protestantismus zum Katholizismus als eigenständiges „Übergangsritual“, das einem Angehörigen der zeitgenössischen Eliten früh eine Verankerung innerhalb der höchsten Spitzen der sich ausbildenden Barockgesellschaft ermöglichte. Sofern es um die grundlegende geographische Verankerung seiner Forschungen geht, leitet sich diese vom Titel eines Aufsatzes Winkelbauers über die Liechtenstein als grenzüberschreitendem Adel ab. Thomas Winkelbauer gehört zu jenen österreichischen Historikern, die ihre Forschungen nicht allein auf das Territorium der heutigen Republik Österreich oder die historischen Gebiete der österreichischen Länder beschränken (österreichische Erblande), sondern die diese Grenzen flächenmäßig, heuristisch und methodologisch mit Blick auf die Ausformung eines synthetisierenden Bildes der Geschichte und Strukturen der habsburgischen Donaumonarchie überschreiten. Thomas Winkelbauer ist dies in bewundernswerter Weise in seinen „Zwillingen“ gelungen, d. h. in der zweibändigen österreichischen Geschichte in der Frühen Neuzeit. Wenngleich es 
sich um eine Synthese der österreichischen Geschichte handelt, wird im Personenregister deutlich, dass in diesem breit aufgefassten Werk die Liechtenstein eine einzigartige Rolle spielen.

Welches Bild des Hauses Liechtenstein hat Thomas Winkelbauer in seinem bisherigen Werk geformt? Bereits in seinem grundlegenden Aufsatz „Die Liechtenstein als grenzüberschreitendes Adelsgeschlecht" aus der Mitte der neunziger Jahre sowie neuerlich in den entsprechenden Teilen des Buches „Liechtensteinisch-tschechische Beziehungen in Geschichte und Gegenwart: Synthesebericht der Liechtensteinisch-Tschechischen Historikerkommission" ordnete Thomas Winkelbauer das Haus Liechtenstein in zwei sehr wichtige historische Kontexte ein - einen zeitlichen und einen räumlichen. Winkelbauer weist auf die Tatsache hin, dass die Liechtenstein im Grenzgebiet zwischen Niederösterreich und Südmähren im 16. Jahrhundert keineswegs aus dem Nichts in Erscheinung kamen, sie sich hier vielmehr bereits seit dem Mittelalter langfristig ihre Machtbasis geschaffen hatten, und zwar in Diensten der österreichischen Erzherzöge und böhmischen Könige. Er verfolgt deren Geschichte seit dem ausgehenden 11. Jahrhundert, als die späteren Herren von Liechtenstein als Lehnsmannen der Vohburger und seit der Mitte des 13. Jahrhunderts, als hier der „Urahn“ Hugo von Liechtenstein-Petronell in Erscheinung trat, nachweisbar sind. Aus der genannten Charakteristik geht hervor, dass Winkelbauer die Liechtenstein nicht allein als althergebrachtes Geschlecht betrachtet, das später gerade aus dieser Altertümlichkeit seine hohen Ambitionen abzuleiten vermochte, sondern zugleich als ein traditionell mit den europäischen Herrscherdynastien und deren Höfen verbundenes Geschlecht. Eine zweite wichtige Beobachtung Winkelbauers ist die Charakteristik der Liechtenstein als "grenzüberschreitendes Adelsgeschlecht". Winkelbauer hat sorgfältig dargelegt, wie die erworbenen (unter konkreten Bedingungen auch verloren gegangenen) Güter entlang der österreichisch-mährischen Grenze langsam wuchsen, was es den Liechtenstein früher als anderen Adelsfamilien ermöglichte, zu Angehörigen mehrerer Ständegemeinden aufzusteigen. Auch wenn die Liechtenstein nach einigen Jahrhunderten noch immer im Schatten der bedeutsameren Aristokratenfamilien verblieben, sollte deren Altertümlichkeit und geographische Ausbreitung doch die Voraussetzung für ihren späteren gesellschaftlichen Aufstieg bilden. Das erwähnte räumliche Prinzip in der Geschichte der Familie Liechtenstein hängt unmittelbar mit deren Besitz- und wirtschaftlichen Aktivitäten zusammen. Vor allem in den achtziger Jahren des 20. Jahrhunderts, als sich Winkelbauer intensiv dem Studium der Wirtschaftsgeschichte und der Geschichte nichtprivilegierter Schichten widmete, konnte er in einem breiten Kontext aufzeigen, wie sich die Liechtenstein in der Langzeitperspektive um die Grundsteinlegung ihres familiären Aufstiegs mit Blick auf den Grundbesitz bemühten. Offenkundig ging gerade von hier deren neuzeitliches Interesse an einer Kultivierung der Landschaft, der Landwirtschaft und des Gartenbaus aus, aber auch an den Anfängen des Tourismus in romantischer Landschaft. Winkelbauer konnte darüber hinaus in seinen Untersuchungen aufzeigen, dass der Grundbesitz für die Liechtenstein in der Frühen Neuzeit nicht allein eine Immobilie darstellte, sondern dass dieser zugleich die Beziehungen zwischen den Menschen - das Verhältnis zwischen Obrigkeit und deren Untertanen eingeschlossen - widerspiegelte. Winkelbauer hat dies beispielsweise in seiner Untersuchung 
über das Verhältnis der Liechtenstein zu den im Verlaufe des 16. Jahrhunderts auf deren südmährischen Gütern sich niedergelassenen Wiedertäufern dokumentiert.

Das Werk Thomas Winkelbauers über das Haus Liechtenstein fokussiert sich auf zwei Persönlichkeiten, die an der Schwelle ihres Aufstiegs in die Kategorie der Fürsten stehen. Karl I. von Liechtenstein widmete sich Winkelbauer mehr oder weniger am Rande, dessen ungeachtet ist er sich über dessen ungewöhnlicher Bedeutung sowohl für den eigentlichen Zeitraum um 1600 als auch für nachfolgende historiographische Einordnung im Klaren. Nicht zuletzt wurde er sich dieser Tatsache bewusst, als er sich mit der Bewertung des bedeutendsten Repräsentanten des Hauses Liechtenstein aus der Feder Roman Vondras befasste. In dem entsprechenden Beitrag reflektiert er das Verhältnis zwischen der Geschichte und deren moderner Aktualisierung und Thematisierung bzw. die Entstehung und das Fortdauern historischer Stereotypen. Seine Interpretation der Persönlichkeit des liechtensteinischen Magnaten baute er dessen ungeachtet auf der Person von Karls jüngstem Bruder, des Fürsten Gundaker von Liechtenstein, auf. In seiner Monographie „Fürst und Fürstendiener" beschreibt er Gundaker in zahlreichen Zusammenhängen: Winkelbauer zeigt zum einen auf, wie vielschichtig die Persönlichkeit des frühneuzeitlichen Magnaten komponiert ist (und dies trotz der Tatsache, dass er sich die Frage stellt, ob wir im Grunde genommen nicht mit Blick auf die Epoche des Übergangs vom 16. zum 17. Jahrhundert von einer „Krise der Aristokratie“ sprechen können). In erster Linie wird diese Persönlichkeit durch das Verhältnis zu Herrscher, Dynastie und Hof definiert, für den Liechtenstein die unterschiedlichsten Gutachten erstellte und dem er mit seinen Ratschlägen diente; hier offenbarten sich jedoch zugleich die verschiedenartigsten Konflikte hinsichtlich des Wirkungsgrades auf der gesellschaftlichen Skala. Den zweiten Wirkungskreis stellt der Aufbau des fürstlichen Hofes dar, was im Falle Gundakers dessen ein wenig aussichtslose Bemühungen um die Gründung eines eigenen (und darüber hinaus Sekundär-) Fürstentums mit den Zentren in Mährisch Kromau (Moravský Krumlov) und Ungarisch Ostra (Uherský Ostrov) darstellten. Es handelte sich um Anstrengungen, die das Haus Liechtenstein gänzlich aus der Gemeinde des mährischen Landesadels ausgegliedert hätten, wobei dieses Bestreben trotz alledem weder bei den mährischen Ständen noch beim Herrscher ein positives Echo finden konnte. Doch auch so schuf sich Gundaker von Liechtenstein als Repräsentant der manieristischen Fürstenschicht einen eigenen Hof mit einem Netzt von Residenzen und einem fürstlichen Zeremoniell. Ein höfisches Milieu, in dem der Fürst Kunst und Musen, höfische Feste sowie tägliche Lustbarkeiten, weltliche Bildung und kirchliche Strukturen förderte. Wenn wir Winkelbauers Monographie über Gundaker von Liechtenstein studieren, können wir nicht die Tatsache außer Acht lassen, dass gerade konfessionelle Fragen im Blickpunkt stehen, was im Grunde genommen das gesamte erste Drittel des Buches ausmacht. Hier wird religiösen Konvertierungen, einem Übergangsritual besonderer Art, Aufmerksamkeit geschenkt, das in einem dramatischen Augenblick der Frühen Neuzeit nicht allein eine konfessionelle, sondern zugleich eine weltliche, politische Bedeutung besaß. Einem zum Katholizismus Konvertierten öffneten sich die Türen in den Schoß der katholischen Kirche und zu politischer Karriere. Die Liechtenstein werden in Winkelbauers Interpretation mit der breiten Gesellschaft 
der mitteleuropäischen Aristokratie konfrontiert und kontextualisiert. Zugleich werden die Liechtenstein nicht allein als ein Adelsgeschlecht an der geographischen Grenze zwischen Österreich und Mähren beschrieben, sondern auch als aristokratische Familie zwischen Reformation und Gegenreformation. Winkelbauer zeigt auf, dass auch im Rahmen der neuen, durch die Trias der Liechtenstein-Brüder gebildeten Familiengeneration eine beachtliche Pluralität zu finden ist. Karl I. von Liechtenstein sieht der Autor als weltlichen Magnaten, der die eigene Konvertierung eher als weltlichen Akt betrachtet. Maximilian von Liechtenstein ist ein Mann zwischen Armee und Kirche, zwischen Schwert und Altar. Er ist einer der kaiserlichen Offiziere am Weißen Berg, zugleich aber auch Gründer des Paulaner-Klosters mit dem liechtensteinischen Familiengrab in Wranau (Vranov u Brna). Gundaker von Liechtenstein wiederum erscheint als ein Mann mit einer intensiven Beziehung zur Theologie, als Leser der Schriften von Valerian Magni und Unterstützer des Piaristenordens. Alle drei Brüder Liechtenstein bilden somit eine Einheit in der Vielfalt und verkörpern das „konfessionelle Zeitalter“. Zugleich stehen sie mit ihrer katholischen religiösen Verankerung an der Schwelle einer bedeutenden Familientradition.

Wie aus Winkelbauers Synthese „Ständefreiheit und Fürstenmacht“ ersichtlich wird, haben sich die Liechtenstein in großem Maße als Mitbegründer der frühneuzeitlichen Habsburgermonarchie betätigt. Nicht allein im Verhältnis zu den Angehörigen der herrschenden Habsburgerdynastie, sondern auch in bedeutendem Maße mit Blick auf die Liechtenstein lässt sich einer der Termini des Buchtitels - „Fürstenmacht“ - anwenden. Die Liechtenstein erscheinen als Geschlecht, das im Untersuchungszeitraum zahlreiche Grenzen überwand, beispielsweise jene zwischen der Reformation in der Ära vor der Schlacht am Weißen Berg und der Zeit der Gegenreformation nach 1620, zwischen einem ständisch verankerten mittelalterlichen Adelsgeschlecht und einem neuzeitlichen Fürstenhaus. Zwischen „Karrieristen“ und „frommen Männern“.

Die wichtigsten Publikationen Thomas Winkelbauers zum Thema Geschichte des Hauses Liechtenstein:

WINKELBAUER, Thomas: Fürst und Fürstendiener. Gundaker von Liechtenstein (1580-1658), ein österreichischer Aristokrat des konfessionellen Zeitalters. Wien 1997. (Habilitationsschrift.)

WINKELBAUER, Thomas: Fürst und Fürstendiener: Gundaker von Liechtenstein, ein österreichischer Aristokrat des konfessionellen Zeitalters. Wien - München 1999.

WINKELBAUER, Thomas: Österreichische Geschichte 1522-1699. Ständefreiheit und Fürstenmacht. Teil 1, 2. Wien 2003.

WINKELBAUER, Thomas: Gundaker von Liechtenstein als Grundherr in Niederösterreich und Mähren. Normative Quellen zur Verwaltung und Bewirtschaftung eines Herrschaftskomplexes und zur Reglementierung des Lebens der Untertanen durch einen adeligen Grundherrn sowie zur Organisation des Hofstaates und der Kanzlei eines „Neufürsten“ in der ersten Hälfte in der ersten Hälfte des 17. Jahrhunderts. Wien - Köln - Weimar 2008.

GEIGER, Peter - KNOZ, Tomáš - FUČ́IIKOVÁ, Eliška - HORÁK, Ondřej - HOREL, Catherine KRÄFTNER, Johann - ŽUPANIČ, Jan - WINKELBAUER, Thomas: Liechtensteinisch-tschechische 
Beziehungen in Geschichte und Gegenwart: Synthesebericht der Liechtensteinisch-Tschechischen Historikerkommission. Vaduz 2014.

WINKELBAUER, Thomas: Repräsentationsleben, Hofstaat und Hofzeremoniell der Herren bzw. Fürsten von Liechtenstein in der ersten Hälfte des 17. Jahrhunderts. In: BŮŽEK, Václav (Hg.): Život na dvorech a v rezidenčních městech posledních Rožmberků. Opera Historica 3. České Budějovice 1993, S. 179-198.

WINKELBAUER, Thomas: Das „Fürstentum Liechtenstein“ in Südmähren und Mährisch Kromau (bzw. Liechtenstein) als Residenzstadt Gundakers von Liechtenstein und seines Sohns Ferdinand. In: BŮŽEK, Václav (ed.): Život na dvorech barokní šlechty. Opera historica 5. České Budějovice 1996, S. 309-334.

WINKELBAUER, Thomas Karrieristen oder fromme Männer? Adelige Konvertiten in den böhmischen und österreichischen Ländern um 1600. In: CHOCHOLÁČ, Bronislav - JAN, Libor - KNOZ, Tomáš (Hg.): Nový Mars Moravicus. Brno 1999, S. 431-452.

WINKELBAUER, Thomas Karrieristen oder fromme Männer? Adelige Konvertiten in den böhmischen und österreichischen Ländern um 1600. Frühneuzet-Info 10, 1999, Heft 1-2, S. 9-20.

WINKELBAUER, Thomas: Karl von Liechtenstein und das „Prager Blutgericht" vom 21. Juni 1621 als tschechischer Erinnerungsort im Spiegel der Historiographie. In: Liechtensteinisch-Tschechische Historikerkommission (Hg.): Liechtensteinische Erinnerungsorte in den böhmischen Ländern. Vaduz 2012, S. 51-71.

WINKELBAUER, Thomas: Haklich und der Korruption unterworfen. Die Verwaltung der liechtensteinischen Herrschaften und Güter im 17. und 18. Jahrhundert. In: OBERHAMMER, Evelin (Hg.): Der ganzen Welt ein Lob und Spiegel. Das Fürstenhaus Liechtenstein in der frühen Neuzeit. Wien - München 1990, S. 86-114.

WINKELBAUER, Thomas: Herren und Holden. Die niederösterreichischen Adeligen und ihre Untertanen im 16. und 17. Jahrhundert. In: KNITTLER, Herbert (Hg.): Adel im Wandel. Rosenburg 1990, S. 73-79.

WINKELBAUER, Thomas: Der Adel in Ober - und Niederösterreich in der frühen Neuzeit. Versuch eines Literatutüberblicks (seit etwa 1950). In: BƯŽEK, Václav (Hg.): Spojující a rozdělující na hranici. Verbindendes und Trennendes an der Grenze. Opera Historica 2. České Budějovice 1992, S. 13-33.

WINKELBAUER, Thomas: Wandlungen des mährischen Adels um 1600. In: MACK, Karkheinz (Hg.): Jan Amos Comenius und Politik seiner Zeit. Wien - München 1992, s. 16-36.

WINKELBAUER, Thomas: Krise der Aristokratie? Zum Strukturwandel des Adels in den böhmischen und niederösterreichischen Ländern im 16. und 17. Jahrhundert. Mitteilungen des Instituts für österreichische Geschichtsforschung 100, 1992, S. 328-353.

WINKELBAUER, Thomas: Überkonfessionelles Christentum in der 2. Hälfte des 16. Jahrhundert in Mähren und seinen Nachbarländern. In: CHOCHOLÁČ, Bronislav - JAN, Libor - KNOZ, Tomáš - MALÍr, Jiří (Hg.): Dějiny Moravy a Matice moravská. Problémy a perspektivy. Brno 2000, S. 131-146. 


\section{Zusammenfassung \\ Thomas Winkelbauer a výzkum dějin Lichtenštejnského knížecího domu}

Profesor Vídeňské univerzity Thomas Winkelbauer přináleží k evropsky uznávaným reprezentantům výzkumu raného novověku. Při pohledu na jeho bibliografii je přitom patrné, že značnou část své badatelské aktivity věnoval knížecímu rodu Lichtenštejnů, a to jednak monograficky (především osobnostem Gundakera z Lichtenštejna a Karla z Liechtensteina), ale také kontextuálně (v rámci dějin šlechty v raném novověku i v rámci široce pojatých rakouských dějin). Thomas Winkelbauer napsal významnou monografii o Gundakeru z Lichtenštejna, kterou později ještě doplnil dalšími speciálními pracemi a také edicemi pramenů. Na př́kladu tohoto rakousko-moravského aristokrata ukazuje, jakým zpo̊sobem svět aristokracie v raném novověku fungoval. Zároveň se dostává k tématu konverzí a náboženství jako jednoho z rozhodujících fenoménů, který v raném novověku bezprostředně souvisel s politickým vzestupem jedince i jeho rodu. Nelze zapomenout ani to, že pro Winkelbauera Lichtenštejnové představují „šlechtu neznající hranici“. Všechny uvedené skutečnosti lze potom postihnout i ve Winkelbauerově významné syntéze rakouských dějin v 16. a 17. století. 

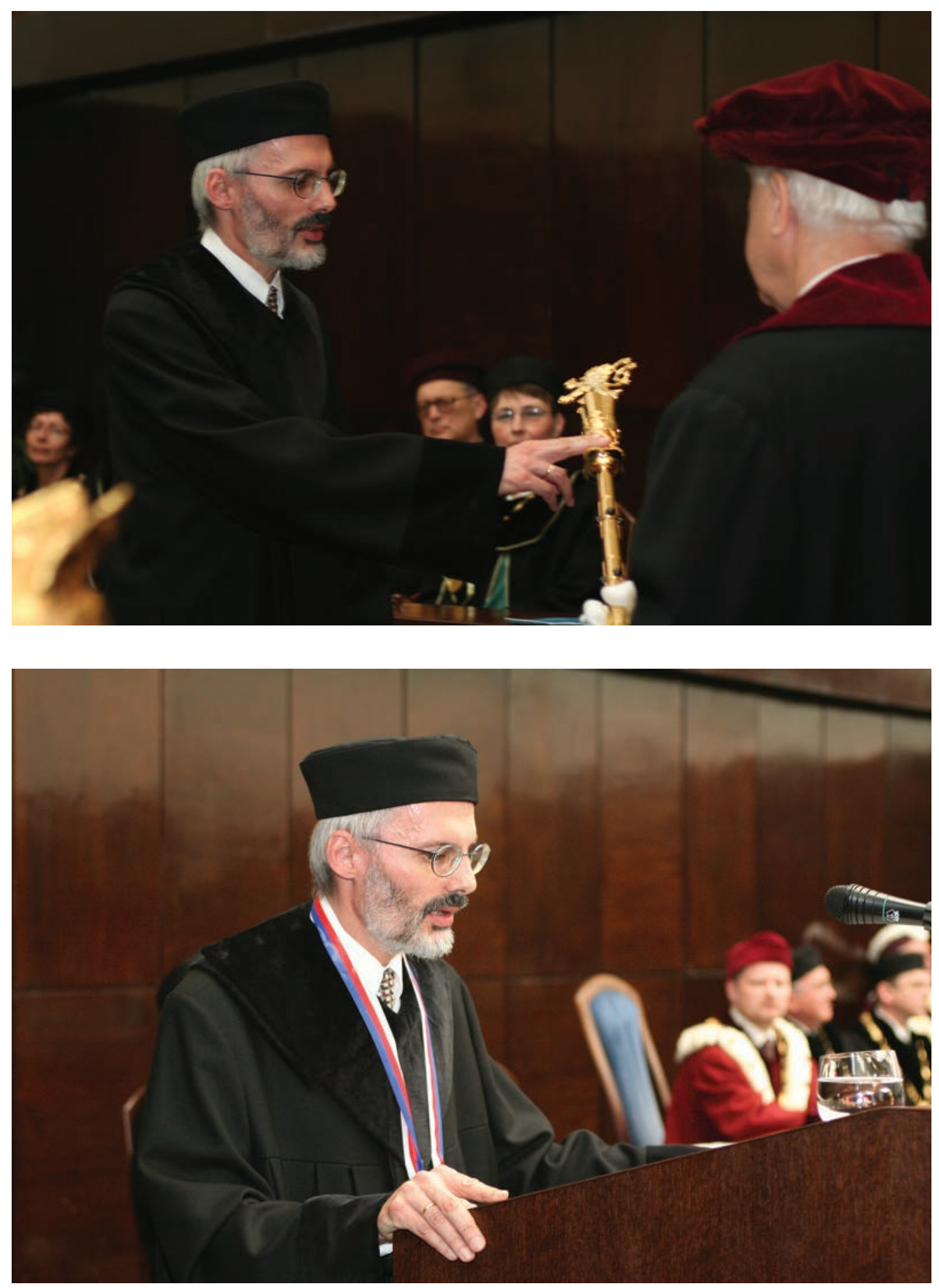